\title{
Análisis territorial del impacto de la Revisión Tarifaria Integral eléctrica de 2016 en el sector residencial argentino: el caso de la ciudad de La Plata, Buenos Aires (2014-2018)
}

\author{
Pedro Chévez ${ }^{1}$ | Irene Martini ${ }^{2}$ | Carlos Discoli ${ }^{3}$ \\ Recibido: 01/03/2020 | Aceptado: 30/06/2020
}

\section{Resumen}

La Revisión Tarifaria Integral (RTI) de electricidad llevada a cabo en 2016 en Argentina ha generado un impacto significativo en la sociedad, resultando en incrementos del orden del $2000 \%$ (nominales) y 1000\% (reales) en las facturas residenciales (2014 vs. 2018). Esto generó un consecuente incremento en la participación del costo del servicio eléctrico sobre los ingresos familiares, el cual tuvo un impacto diferenciado en los distintos estratos sociales. En efecto, surgen interrogantes acerca de cuál fue el comportamiento energético (incremento/decrecimiento) de los distintos usuarios ante un contexto de fuertes aumentos en el precio de este vector energético. En este sentido, el artículo plantea un análisis territorial de las variaciones del consumo eléctrico residencial entre 2014 y 2018 en la ciudad de La Plata (Buenos Aires-Argentina), en dos escalas de trabajo: la intra-urbana (cinco áreas homogéneas) y la global-urbana (total municipio). Este abordaje permite identificar comportamientos diferenciados y extraer conclusiones respecto del impacto territorial de la Revisión Tarifaria Integral. Los principales resultados demuestran que en la escala global-urbana el consumo fue de $-3,33 \%$; mientras que a escala intra-urbana se encuentran variaciones que van desde $-12,84 \%$ hasta $+17,51 \%$, las cuales están directamente asociadas a variables socio-económicas y de consolidación que caracterizan a cada área homogénea.

Palabras clave: demanda eléctrica; tarifas; sector residencial; consumo; energía.

\begin{abstract}
Territorial analysis of the 2016 electrical Integral Tariff Review impact in the Argentinian residential sector: the case of La Plata city, Buenos Aires (2014-2018)

The electricity Integral Tariff Revision (ITR) carried out in 2016 in Argentina has generated a significant impact on society, resulting in increases of $2000 \%$ (nominal) and $1000 \%$ (real) in residential bills (2014 vs. 2018). This situation generated a consequent increase in the share of the cost of electric service over family income, which had a differentiated impact over each social stratum.

1. Instituto de Investigaciones y Políticas del Ambiente Construido. Consejo Nacional de Investigaciones Científicas y Técnicas Universidad Nacional de La Plata. E-mail: che.pedro@hotmail.com

2. Instituto de Investigaciones y Políticas del Ambiente Construido. Consejo Nacional de Investigaciones Científicas y Técnicas Universidad Nacional de La Plata. E-mail: irenemartini@conicet.gov.ar

3. Instituto de Investigaciones y Políticas del Ambiente Construido. Consejo Nacional de Investigaciones Científicas y Técnicas Universidad Nacional de La Plata. E-mail: discoli@rocketmail.com
\end{abstract}


Indeed, questions arise about the energy behavior (increase/decrease) of the different users in the context of strong increases in the price of this energy vector. Hence, we present a territorial analysis of the variations in residential electricity consumption between 2014 and 2018 in La Plata city (Buenos Aires-Argentina), in two work scales: an intra-urban (five homogeneous areas) and a global-urban (total municipality). This approach allows identifying differentiated behaviors and getting conclusions regarding the territorial impact of the Integral Tariff Revision. The main results show that in the global-urban scale consumption was $-3.33 \%$; while in the intra-urban scale there are variations ranging from $-12.84 \%$ to $+17.51 \%$, which are associated with socio-economic and consolidation variables that characterize each homogeneous area.

Keywords: electricity demand; tariffs; residential sector; consumption; energy.

\section{Introducción}

A nivel global la energía es considerada como un factor fundamental para el desarrollo socio-económico de los países; sin embargo, según el tratamiento que reciba por parte de los gobiernos ésta puede ser concebida como un bien estratégico -social- o como un bien de intercambio-commodity-. En base a dichas consideraciones, una de las variables en las cuales se refleja directamente esta orientación es la del precio fijado para su comercialización. En consecuencia, para el caso particular de las últimas décadas en Argentina, desde los años posteriores a la crisis económicosocial de 2001 hasta la actualidad, es posible identificar dos períodos bien diferenciados en términos de política tarifaria energética, los cuales se enuncian brevemente a continuación.

El primer período comienza con la sanción de la Ley de Emergencia Pública y Reforma del Régimen Cambiario (ley No 25.561/02) durante el gobierno provisional de Eduardo Duhalde (20022003). Esta ley implicó, entre otros aspectos, el congelamiento y la desdolarización de las tarifas de gas natural y de electricidad de las empresas con jurisdicción nacional -éstas se localizan en el Área Metropolitana de Buenos Aires (AMBA) y prestan servicio a un 35\% de los usuarios totales a nivel nacional-. En los años posteriores, durante el gobierno de Néstor Kirchner (2003-2007), ante el inicio de un proceso inflacionario y de devaluación del peso argentino, se manifestó un incipiente desajuste en los ingresos y egresos de las distribuidoras energéticas. Por consiguiente, el gobierno optó por la incorporación de un sistema de subsidios al consumo de carácter universal para todos los usuarios del AMBA. Estos subsidios, a su vez, funcionaban como un aumento indirecto del ingreso económico y fomentaba la reactivación del sector industrial. Posteriormente, bajo la administración de Cristina Fernández (2007-2015), el mencionado proceso inflacionario y de devaluación se intensificó y, por consiguiente, se necesitaron cada vez más recursos del Tesoro nacional para cubrir tanto la brecha entre la recaudación de las distribuidoras y sus costos operativos, como así también los desajustes provocados en la cadena de pagos de los restantes sectores de la industria energética. En este sentido, hacia 2011, el gobierno intentó comenzar a desarmar este sistema, promoviendo la renuncia voluntaria a los subsidios por parte de los usuarios, luego eliminándolos en zonas características con alto poder adquisitivo $\mathrm{y}$, finalmente, en 2014 reduciéndolos de manera prácticamente generalizada en un valor promedio del $20 \%$.

El segundo período está signado por el cambio de gobierno en diciembre de 2015, cuando asume la presidencia Mauricio Macri (2015-2019). Esta nueva administración optó por realizar una Revisión Tarifaria Integral (RTI) en 2016, dos meses después de asumir la presidencia. Esta revisión había sido postergada desde 2002. El objetivo era eliminar definitivamente los subsidios al consumo de gas natural y electricidad, recomponer la cadena de pagos y fijar nuevos valores 
para los costos de producción para, de esa manera, reducir el déficit fiscal. En este sentido, por ejemplo, la Resolución 6/2016 sostiene que era necesario modificar el esquema previo de tarifas eléctricas dado que:

(...) el abandono de criterios económicos en la definición de los precios del Mercado Eléctrico Mayorista (MEM) distorsionó las señales económicas, aumentando el costo de abastecimiento, desalentando la inversión privada de riesgo dirigida a incrementar eficientemente la oferta y restando incentivos al ahorro y el uso adecuado de los recursos energéticos por parte de los consumidores y usuarios. (Infoleg, 2019).

Tal como era previsible, los fuertes cambios introducidos en 2016 en materia de políticas tarifarias energéticas generaron un impacto significativo en la sociedad. La Revisión Tarifaria Integral (RTI) generó incrementos promedio del precio de la energía del orden del $2000 \%$ (nominales) y $1000 \%$ (reales) en las facturas residenciales ${ }^{4}$, considerando los años 2014 y 2018 como referencia. El mencionado incremento nominal del $2000 \%$, a su vez, puede ser analizado de forma desagregada por tipos de usuarios. La Tabla 1 muestra los resultados del cálculo del costo de la factura eléctrica de diez tipos de usuarios, con valores de consumo predeterminados que van desde 300 a $2500 \mathrm{kWh} /$ bimestre, considerando los cuadros tarifarios de 2014 y de 2018 (sin impuestos). Allí se puede observar que los usuarios con mayores aumentos fueron aquellos que consumen 600, 750 y $900 \mathrm{kWh} /$ bimestre, con incrementos de 3692\%, 4516\% y 3898\%; luego los usuarios de menores consumos, 300 y $450 \mathrm{kWh} /$ bimestre, presentaron incrementos del 2269\% y 2734\%; los usuarios con altas demandas, 1050,1200 y $1350 \mathrm{kWh} /$ bimestre, presentaron aumentos del 1796\%, $1791 \%$ y $1577 \%$; mientras que los de máximo consumo, 1500 y $2500 \mathrm{kWh} /$ bimestre, contaron con aumentos del 1135\% y 1094\%. En efecto, la reforma tarifaria apuntó a eliminar subsidios, incrementar los precios de la energía y restituir la cadena de pagos facilitando a las distribuidoras la recaudación directa a través de los usuarios; lo cual, tal como se señaló previamente, se realizó por medio de una redistribución desigual y cuyo mayor ajuste recayó mayormente sobre los sectores de consumo medio y bajo, quienes constituyen el grueso de los usuarios. Por su parte, los consumidores que contaban con altas demandas sufrieron las menores consecuencias de esta política.

En consecuencia, la RTI representó un drástico cambio en la incidencia de los servicios energéticos sobre los ingresos familiares; por lo cual, surgen interrogantes en torno al comportamiento de los usuarios residenciales respecto de la demanda eléctrica ante un contexto de importantes incrementos tarifarios. Como aproximación inicial, es posible analizar la evolución de la demanda residencial -neta y per cápita ${ }^{5}$ - en Argentina, entre 2005-2018 (Figura 1). Allí se observa que tanto la demanda neta como per cápita fueron crecientes entre 2005-2015, para luego presentar una marcada caída en 2016-2017-2018.

4. Se entiende por valor nominal al precio corriente de cada año considerado, mientras que el valor real tiene en cuenta la inflación del período estudiado.

5. La demanda residencial per cápita es el cociente entre la demanda residencial neta y la totalidad de habitantes en un determinado año. No se considera la tasa de electrificación de hogares ya que es un dato que no se conoce con desagregación anual. 
Tabla 1. Comparativa de pago por el servicio eléctrico (sin impuestos) en 2014 y 2018 utilizando el cuadro tarifario de EDELAP S.A.

\begin{tabular}{|c|c|c|c|}
\hline $\begin{array}{l}\text { Consumo } \\
\text { Bimestral } \\
\text { [kWh/bim] }\end{array}$ & $\begin{array}{c}\text { Pago } 2014 \\
\text { [AR\$/bim] }\end{array}$ & $\begin{array}{l}\text { Pago } 2018 \\
\text { [AR\$/bim] }\end{array}$ & Aumento [\%] \\
\hline 300 & 38,06 & 863,46 & $2269 \%$ \\
\hline 450 & 50,81 & 1389,32 & $2734 \%$ \\
\hline 600 & 59,51 & 2197,32 & $3692 \%$ \\
\hline 750 & 74,21 & 3351,27 & $4516 \%$ \\
\hline 900 & 97,41 & 3797,37 & $3898 \%$ \\
\hline 1050 & 236,21 & 4243,47 & I796\% \\
\hline 1200 & 261,86 & 4689,57 & I79I\% \\
\hline 1350 & 325,76 & 5135,67 & $1577 \%$ \\
\hline 1500 & 514,26 & 5837,25 & $1135 \%$ \\
\hline 2500 & 807,26 & 8830,65 & $1094 \%$ \\
\hline
\end{tabular}

Fuente: elaboración propia en base a cuadros tarifarios de la distribuidora local (EDELAP S.A.).

Figura 1. Evolución del consumo residencial argentino de energía eléctrica neta y por persona.

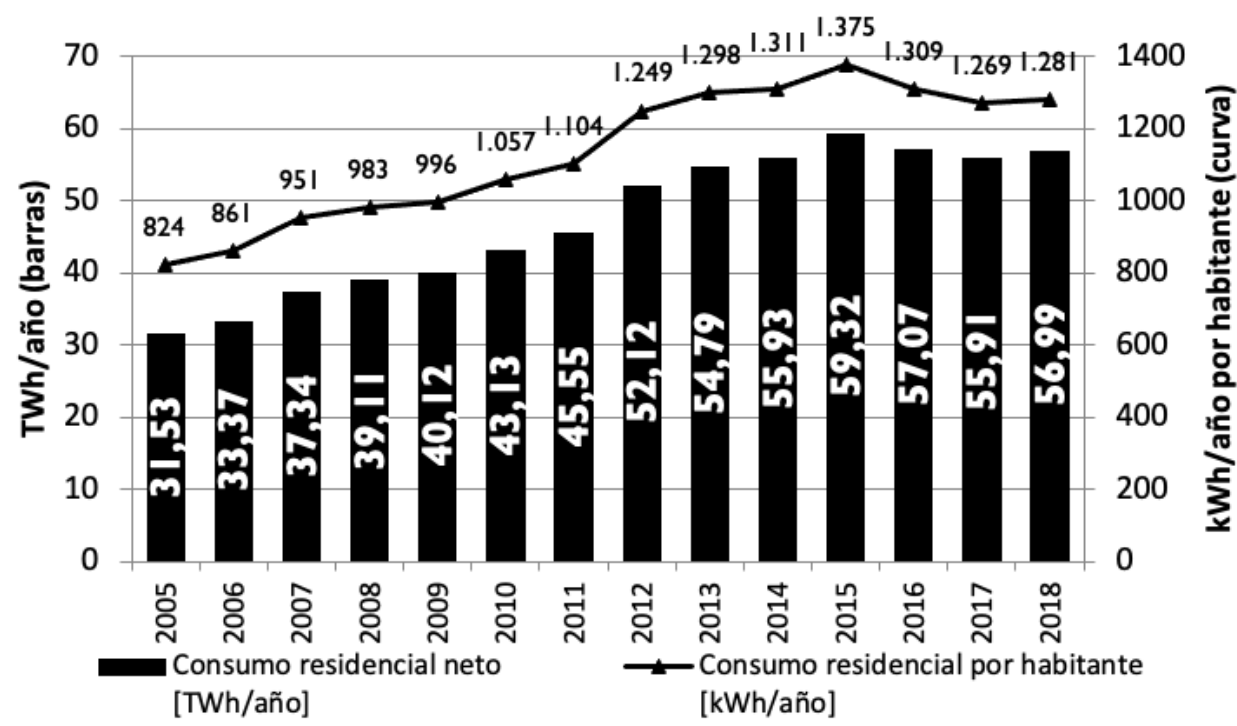

Fuente: elaboración propia en base a información estadística de Cammesa (2019) y proyecciones de población de INDEC (2019).

En efecto, la demanda residencial per cápita entre 2005-2015 demostró un incremento de $+66 \%$, pasando de 824 a $1375 \mathrm{kWh} /$ año por habitante, principalmente por la reactivación económica ocurrida luego de la salida de la crisis de 2001 que permitió la incorporación de electrodomésticos en las viviendas. Esto, a su vez, estuvo acompañado por un esquema de tarifas y subsidios que representaban una baja incidencia de la canasta energética sobre el ingreso total de los hogares. Para graficar la inserción de electrodomésticos a las viviendas, es posible citar a modo de ejemplo que en 1996 un 6\% de los hogares contaba con microondas; un 36\% tenía heladera con freezer; un $7 \%$ tenía computadora y un $4 \%$ contaba con aire acondicionado. Mientras que en 2012, los mencionados valores ascendieron a $37 \%$ (microondas); $74 \%$ (heladera con freezer); $52 \%$ (com- 
putadora) y 34\% (aire acondicionado) (Chévez, 2018; INDEC, 2018a; 2018b). En relación a dicho aspecto, es preciso destacar la importancia de la aplicación del sistema de etiquetado de electrodomésticos, la implementación de estándares mínimos para la comercialización de equipos, el reemplazo y prohibición de ventas de lámparas incandescentes, entre otras medidas; puesto que, de lo contrario, el incremento en la demanda de electricidad residencial por persona hubiera superado ampliamente el $+66 \%$ mencionado previamente. En contraposición, entre 2016 y 2018 la demanda residencial por persona comenzó a mostrar una tendencia decreciente, alcanzando un valor de $1281 \mathrm{kWh} /$ año per cápita en 2018 (-7\% respecto de 2015), lo cual denota la incidencia de los nuevos cuadros tarifarios impuestos en 2016. En relación a esto, es preciso destacar que no hubo innovaciones ni políticas energéticas significativas en términos de eficiencia energética, sino que se mantuvieron las medidas enunciadas para el período anterior.

Si analizamos la demanda neta residencial, es decir la totalidad de la energía eléctrica consumida en los hogares argentinos, ésta pasó de 31,5 TWh/año en 2005 a 59,32 TWh/año en 2015 (+88\%). Este crecimiento se explica tanto por el crecimiento vegetativo de la población como por el mencionado incremento en la demanda per cápita. Posteriormente, la demanda total de electricidad residencial cayó en los años sucesivos alcanzando los 56,99 TWh/año en 2018 (-4\% respecto de 2015), a pesar de que la población continuó en aumento.

A partir de este análisis inicial, es posible verificar que desde 2016 la demanda de energía eléctrica residencial (neta y per cápita) fue decreciente. Sin embargo, este análisis agregado no permite identificar variaciones territoriales, en las cuales pueden originarse comportamientos diversos tales como decrecimientos de variada intensidad o incluso incrementos en la demanda. A pesar de la importancia de este tipo de aproximaciones territoriales, los estudios de energía en Argentina por lo general se orientan a trabajar con valores agregados, perdiendo de vista aspectos tales como el emplazamiento urbano, el clima, condiciones socio-demográficas, etc., los cuales pueden proveer información para comprender dichas diferenciaciones. Esta situación conlleva a perder de vista un elemento importante, que es el del direccionamiento de las políticas, las cuales indefectiblemente deberán diferenciarse en el territorio. Por consiguiente, el presente trabajo plantea como hipótesis que: los estudios con anclaje territorial permiten profundizar y comprender de manera más precisa los procesos analizados bajo enfoques globales y, por consiguiente, pueden ser de utilidad para el diseño de políticas públicas.

\subsection{Revisión de literatura e identificación del vacío en las investigaciones}

Dada la celeridad con que el gobierno de Mauricio Macri presentó e implementó las recientes transformaciones en materia de política económica y energética, su cercanía temporal, como así también lo coyunturales que resultan sus consecuencias, aún no es posible identificar estudios científicos que hayan abordado el impacto de los mencionados cambios sobre la sociedad. Sobre la mencionada temática, únicamente es posible identificar informes ofrecidos por universidades, consultoras, empresas, etc., en los cuales se abordan aspectos puntuales.

Por ejemplo, el informe de la Universidad Nacional de Avellaneda (Fraschina, 2018a) indaga sobre el aumento en el peso de los servicios públicos sobre el salario mínimo entre 2015-2018, concluyendo que Argentina es el país de la región que mayor incremento sufrió en este indicador en el mencionado período; pasando de un 6\% en 2015 a un $21 \%$ en 2018 . Este estudio contempla el peso de los servicios de electricidad, gas natural y agua potable sobre el salario mínimo vital y móvil. Si se considera el transporte público sumado a los servicios mencionados previamente, la 
incidencia total de la canasta básica de servicios pasó de un 6,3\% a un 23,5\% del salario mínimo; y de un 2,1\% a un 7,2\% del salario medio formal (Fraschina, 2018b). Por su parte, el documento de Bondorevsky (2016) ofrece un análisis inicial de la RTI de 2016, donde se destaca la dificultad de acceso a la información, metodologías de cálculo y tasas de rentabilidad con las cuales se modificaron los esquemas tarifarios, como así también la imposibilidad de involucrar a los usuarios en los procesos de decisión, entre otros factores. Por otra parte, los trabajos de Durán (2018a; 2018b) analizan temporal (2003-2018) y territorialmente (a escala nacional) el impacto de la RTI sobre el costo del servicio y los consecuentes niveles de pobreza energética en distintas ciudades del país.

Otro tipo de publicaciones que permiten verificar el impacto de los cambios en la política tarifaria son los informes anuales de la Compañía Administradora del Mercado Mayorista Eléctrico (Cammesa, 2019), donde es posible relevar las demandas netas de energía para todo el país y por regiones, sin embargo se trata de informes estadísticos que no pretenden indagar y analizar las variables que determinan dichas variaciones. A su vez, la temática fue puesta en agenda de forma cotidiana en periódicos nacionales y portales de noticias debido a las sucesivas modificaciones implementadas en las políticas energéticas y su consecuente impacto en la sociedad.

Por su parte, en relación a estudios energéticos que abordan diferenciaciones territoriales, es posible mencionar algunos ejemplos orientados a la elaboración de diagnósticos. Por ejemplo, los trabajos realizados para la 2da Comunicación Nacional de la República Argentina de la Convención Marco de las Naciones Unidas sobre Cambio Climático (IDEHAB, 2005) cuantificaron emisiones de gases de efecto invernadero considerando consumos diferenciados en el país. También hay que destacar el trabajo de Margulis (2014), quien elabora un modelo que pondera la incidencia de las distintas variables en el consumo eléctrico nacional y en el cual se tienen en cuenta las regiones geográficas argentinas.

Otros ejemplos son las distintas publicaciones elaboradas por el equipo de investigación al que pertenecen los autores de este trabajo: en Chévez, Discoli y Martini (2017) y en Chévez, Martini y Discoli (2018) se presentan dos análisis sobre el consumo residencial argentino, tanto de gas natural como de electricidad, con el objetivo de comprender la interacción entre variables climáticas, socio-demográficas, equipamiento domiciliario y consumo final en las diferentes provincias del país. Otro ejemplo es el artículo de Chévez, Martini y Discoli (2019a) que se centró en el desarrollo de una metodología para elaborar diagnósticos de energía urbana, utilizando a la ciudad de La Plata como caso de estudio, atendiendo a delimitar áreas homogéneas e identificar sus consumos energéticos, como así también una serie de variables que permiten reconocer y justificar sus patrones de consumo.

En síntesis, a pesar de que el impacto de la Revisión Tarifaria Integral de 2016 es un tema de interés coyuntural y que los impactos globales de su implementación han sido cuantificados por trabajos puntuales, no son frecuentes los estudios que propongan y apliquen desarrollos metodológicos para el análisis de sus diversos efectos en el territorio; en este caso particular, en el ámbito urbano e intra-urbano. 


\subsection{Objetivos y alcances del trabajo}

En base a las problemáticas y al vacío en las investigaciones detectados, el presente trabajo plantea el desarrollo y la aplicación de una metodología para el análisis de las variaciones en el consumo eléctrico residencial de la ciudad de La Plata entre dos años particulares (2014-2018) bajo dos escalas de análisis: (i) la intra-urbana, que aborda la ciudad de forma detallada, desagregándola por áreas homogéneas; y (ii) la global-urbana, que integra la información detallada para toda la ciudad.

Este enfoque tiene por objeto identificar los comportamientos diferenciados de distintas áreas urbanas, las cuales presentan incrementos/decrecimientos de variada intensidad en sus consumos y costos del servicio eléctrico. Para ello, se utilizan cinco áreas referenciales de la ciudad, donde se analiza una muestra representativa de casos (viviendas) para cada una de éstas y se releva una serie de variables e indicadores asociados que posibilitan caracterizarlas de manera general. En tanto, el abordaje desagregado permite integrar la información y obtener un valor único para toda la ciudad respecto de la variación del consumo y el costo del servicio eléctrico.

Este análisis, por consiguiente, pretende indagar sobre los comportamientos de la demanda eléctrica ante un contexto de fuertes incrementos en las tarifas y, además, busca verificar la hipótesis que pondera la importancia de los estudios con anclaje territorial desagregados (en este caso intra-urbanos) ya que sus resultados son capaces de interpelar y profundizar un resultado agregado (global-urbano).

\section{Descripción del caso de estudio}

El presente trabajo desarrolla y aplica una metodología de análisis territorial en un caso de estudio concreto, que es la ciudad de La Plata. No obstante, la misma podría ser replicada en otras ciudades de características similares.

En este sentido, cabe señalar que la ciudad de La Plata es la capital de la provincia de Buenos Aires (Argentina), y que para el año 2014 contaba con una población estimada de 631.831 habitantes. Si bien cuenta con vínculos estrechos con la Región Metropolitana de Buenos Aires, posee una dinámica de funcionamiento propia, definiéndose como una "ciudad intermedia" (Figura 2). Este tipo de ciudades responden a cierta autonomía, pero a su vez son dependientes de las grandes metrópolis, realizan funciones de intermediación entre núcleos más pequeños y grandes áreas metropolitanas, se caracterizan por presentar cierta complejidad funcional, por tener un grado importante de centralidad y poseer significativos elementos de simbología histórica, infraestructura y servicios (Rodríguez, 2016; Soijet et al., 2016). En este sentido, su análisis presenta una gran potencialidad de replicabilidad en universos de similares características, ya que este tipo de ciudades son las de mayor predominancia en Argentina (Discoli, 2009). 
Figura 2: inserción regional del área de estudio. En rojo la región del Gran La Plata, en amarillo el Área Metropolitana de Buenos Aires. Escala: 1: 1.500.000.

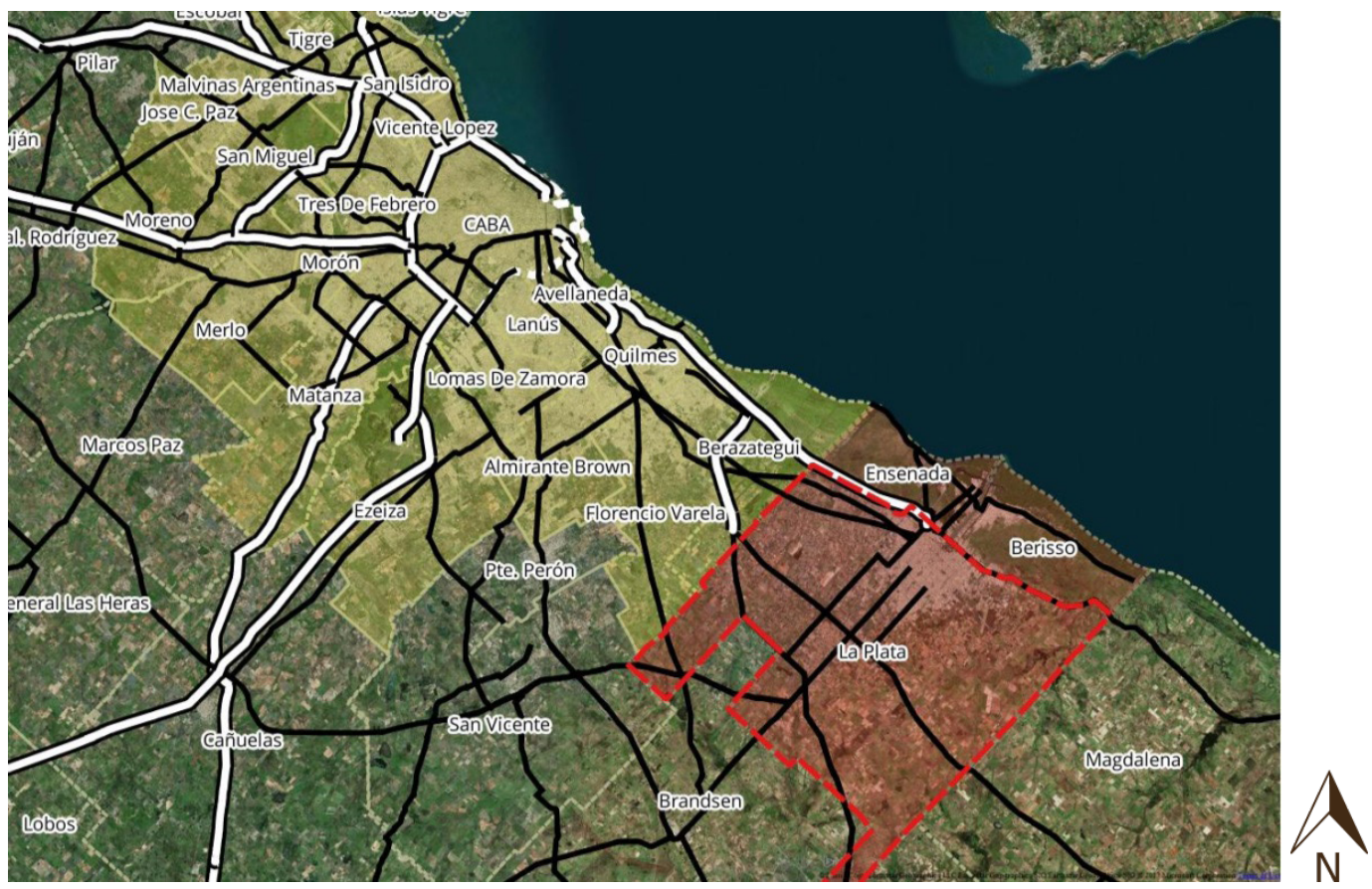

Fuente: Chévez (2018).

\section{Metodología}

Con el objetivo de estudiar y comprender la evolución temporal (2014-2018) del consumo eléctrico residencial en el ámbito urbano (en este caso en la ciudad de La Plata); se propone una metodología que consta de tres etapas. En las dos primeras se utilizan dos escalas territoriales diferentes y en la tercera se realiza la comparativa de sus resultados.

A continuación se describe sintéticamente cada una de ellas, para luego describirlas en profundidad: (i) inicialmente, se aborda la ciudad de forma desagregada, considerando sus diferentes consolidaciones a los efectos de reconocer, en su estructura, unidades de análisis de referencia. Para ello, se utilizan cinco áreas homogéneas $(\mathrm{AH})$, las cuales permitieron subdividir el objeto de estudio y, de esta manera, desarrollar el análisis intra-urbano; (ii) posteriormente, se procede a la integración de las partes para reconstruir el objeto de estudio. Así, se utiliza la ciudad completa como unidad de análisis, pero con la especificidad de sus datos territorializados, lo cual permite emprender el análisis global-urbano; (iii) por último, en la tercera etapa se comparan los resultados de las AHs y de la ciudad completa con el fin de identificar discrepancias/similitudes entre los valores intra-urbanos y el global-urbano.

Por su parte, es preciso aclarar que la selección de los dos años de estudio (2014 y 2018) responde a una situación equivalente, previa y posterior, respecto de la Revisión Tarifaria Integral (RTI) realizada en el año 2016. Es decir que se busca analizar el comportamiento de los usuarios dos años antes y dos años después de dicha revisión.

Asimismo, puesto que la demanda de energía tiene vínculos directos con las condiciones climáticas, se analizaron las temperaturas medias anuales de 2014 y 2018 para la ciudad de La Plata - 
Aeropuerto- (Tu Tiempo, 2019) y, a modo de establecer una segunda validación, también para la Ciudad Autónoma de Buenos Aires (DGEyC-CBA, 2019). En relación a dicho análisis, se identifica que la ciudad de La Plata presentó la misma temperatura media para los dos años estudiados $\left(16,7^{\circ} \mathrm{C}\right)$; por su parte, en la Ciudad Autónoma de Buenos Aires también se obtuvo un mismo valor para los dos años, pero levemente superior al del caso platense $\left(18,6^{\circ} \mathrm{C}\right)$.

Por otro lado, también es preciso mencionar que la demanda de energía tiene un vínculo estrecho con las condiciones socio-económicas de la población. En este sentido, se analizó la tasa de desocupación de la ciudad de La Plata para el año 2014 y 2018. A partir de informes del Instituto Nacional de Estadísticas y Censos se observa que el aglomerado del Gran La Plata pasó de contar con una desocupación de 7,2\% en 2014 (INDEC, 2020a) a 8,33\% en 2018 (INDEC, 2020b). Es decir que la situación del mercado de trabajo empeoró en este intervalo de tiempo, lo cual puede anticipar ciertos resultados globales en cuanto a la evolución de la demanda eléctrica residencial.

A continuación se describen de forma detallada las tres instancias metodológicas, con sus correspondientes niveles de análisis.

\subsection{Abordaje territorial desagregado por áreas homogéneas: nivel de análisis intra- urbano}

Las unidades de análisis, denominadas áreas homogéneas $(\mathrm{AH})$, fueron delimitadas, descriptas y estudiadas en trabajos previos (Chévez, 2018; Chévez et al., 2019a) y, a modo de definición conceptual, se puede afirmar son áreas de la ciudad que presentan características internas similares en cuanto al comportamiento de una serie de variables socio-demográficas y energéticas predefinidas, las cuales se vinculan directamente con los niveles de consolidación urbana. Estas áreas surgen del agrupamiento de radios censales con características censales y energéticas análogas, por medio de la aplicación de un algoritmo de clasificación denominado clustering de k-medias (Chévez et al., 2019a). Las variables utilizadas para la clasificación de radios censales fueron: el promedio de personas por hogar, el promedio de habitaciones por hogar, el porcentaje de hogares con tipología de vivienda "casa", el promedio de hogares por hectárea, el consumo promedio de electricidad ( $\mathrm{kWh} /$ año por usuario) y el consumo promedio de gas natural ( $\mathrm{m}^{3} /$ año por usuario).

La delimitación territorial de las áreas homogéneas se puede verificar en la Figura 3. Allí se identifican cinco áreas: en primera instancia, un AH localizada en el centro del trazado fundacional, es decir, el casco urbano, la cual se denomina AH1-Casco centro; luego, a su alrededor, una segunda AH que se extiende hasta los límites del casco urbano denominada AH2- Casco bordes; posteriormente, se observa un $\mathrm{AH}$ que rodea el trazado fundacional y que, también, abarca sectores intersticiales de la periferia, llamada AH3- Expansión del casco; por otro lado, en el eje noroeste, conformado por las localidades de Gonnet, City Bell y parte de Villa Elisa y M. Romero, se ubica la denominada AH4- Eje noroeste y, en última instancia, se observa un AH que cubre los radios censales periféricos de menor consolidación, llamada AH5- Periurbano. En efecto, cada una de las áreas presenta características bien diferenciadas entre sí, donde fundamentalmente inciden los niveles de consolidación urbana y los valores de consumo eléctricos y de gas natural. 
Figura 3: mapa del caso de estudio en el cual se distingue la cobertura de cada una de las cinco áreas homogéneas en las que fue dividida la ciudad y la geolocalización de los casos relevados.

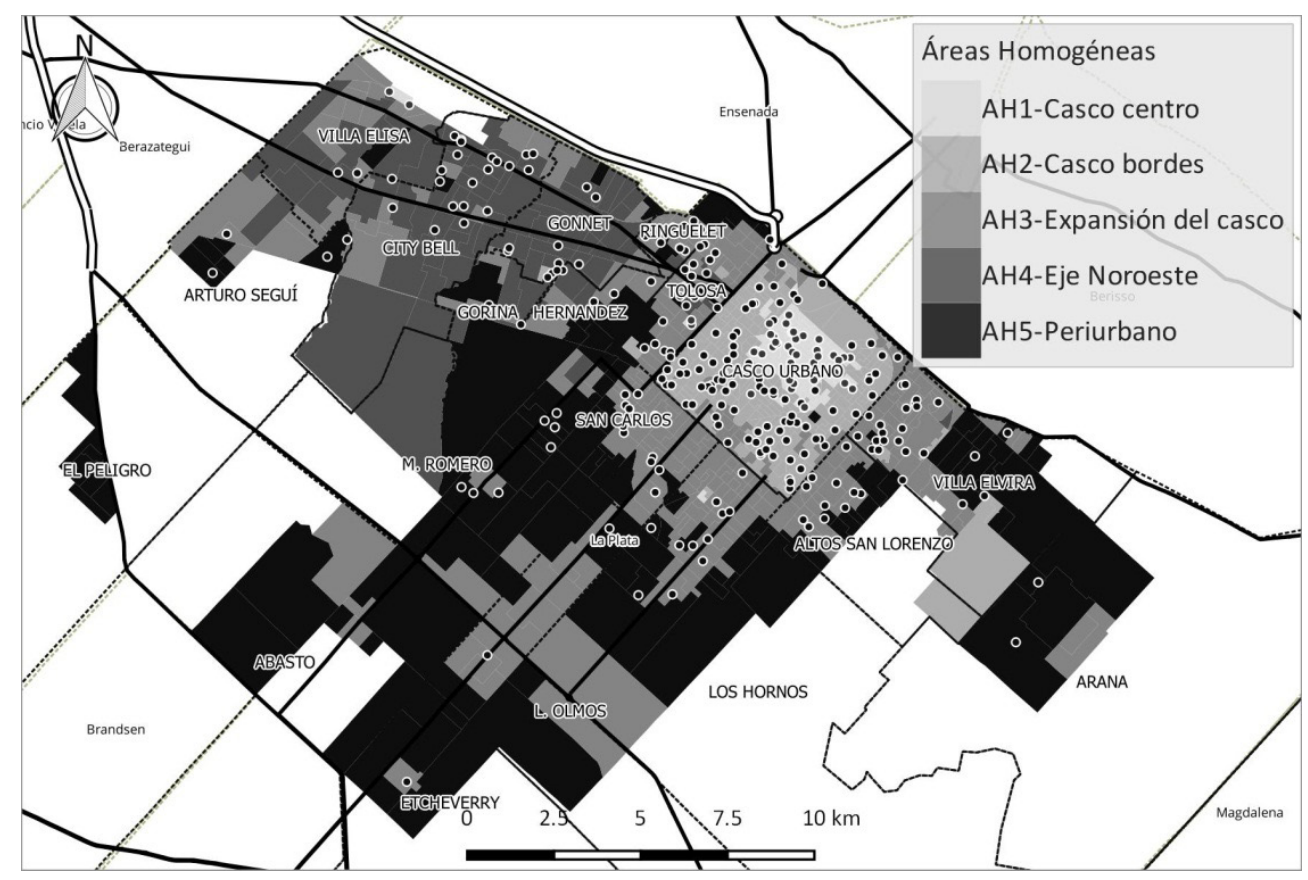

Fuente: Elaboración propia.

Posteriormente, para analizar la variación de la demanda eléctrica en los diferentes sectores característicos de la ciudad se requiere información individualizada del consumo residencial. En consecuencia, para abordar este relevamiento, se calcula la muestra estadística representativa de casos a relevar (viviendas) en cada $\mathrm{AH}$, los cuales permiten calcular los valores medios de consumo y costo del servicio de forma discriminada. A partir del cálculo de la muestra, se seleccionan aleatoriamente viviendas a relevar, a las cuales individualmente se les solicitan dos facturas eléctricas con los consumos anuales de 2014 y de 2018; para ello se requiere la factura del primer bimestre de 2015 y de 2019, ya que de esa manera es posible identificar los consumos completos de 2014 y 2018.

En total se requiere alcanzar los 278 casos relevados (viviendas). Para el cálculo se utilizó la ecuación para muestras estratificadas a partir de la fijación proporcional sin reposición, cuyo desarrollo se puede encontrar en Chévez (2018: 93). El diseño muestral resultante estableció la distribución de casos para cada área homogénea (ver Tabla 2), mientras que la geolocalización de los casos relevados se observa en la Figura 3.

Tabla 2. Cantidad de casos a relevar en cada área.

\begin{tabular}{|c|c|}
\hline Área Homogénea & Casos a relevar \\
\hline $\mathrm{AH} 1$ & 48 \\
\hline $\mathrm{AH} 2$ & 78 \\
\hline $\mathrm{AH} 3$ & 98 \\
\hline $\mathrm{AH} 4$ & 29 \\
\hline $\mathrm{AH} 5$ & 26 \\
\hline Total & 278 \\
\hline
\end{tabular}

Fuente: elaboración propia. 
Una vez relevadas las dos facturas eléctricas de cada caso (vivienda), se identifican y calculan de forma individual los siguientes indicadores:

-Consumo de electricidad en kWh: 6 bimestres (2014), 12 meses (2018) y anual (2014 y 2018). Cabe destacar que en 2014 la factura eléctrica se realizaba de forma bimestral, mientras que para 2018 la misma pasó a ser mensualizada. En consecuencia la sumatoria de estos valores permite totalizar la energía consumida durante cada año de referencia.

- Costo de la electricidad (ARS): 6 bimestres (2014), 12 meses (2018) y anual (2014 y 2018). Para el cálculo del costo bimestral o mensual de la energía, se utilizan los cuadros tarifarios vigentes para cada período de la distribuidora local (véase el Anexo 1).

Por otra parte, es preciso aclarar que para poder realizar comparativas temporales del indicador costo de la energía (AR\$/año) entre 2014 y 2018, ante un contexto inflacionario sostenido, es necesario afectar los valores obtenidos para 2014 a los efectos de actualizarlos y así poder ser comparados con los de 2018 (Ecuación 1).

$$
\mathrm{AR} \$ / \mathrm{anno}_{2018}=\mathrm{AR} \$ / \mathrm{año}_{2014}{ }^{*} \mathrm{I}_{2015}{ }^{*} \mathrm{I}_{2016}{ }^{*} \mathrm{I}_{2017} \quad \text { Ecuación } 1
$$

Dónde:

$\mathrm{AR} \$ / \mathrm{año}_{2018}=$ costo de la energía de 2014 actualizado a 2018

$\mathrm{AR} \$ / \mathrm{año}_{2014}=$ costo de la energía calculado para el año 2014

$\mathrm{I}_{\text {año }}=$ índice de inflación anual: $\mathrm{I}_{2015}=1,243 ; \mathrm{I}_{2016}=1,349 ; \mathrm{I}_{2017}=1,235(\mathrm{DGEyC}-\mathrm{CBA}, 2020)^{6}$.

En síntesis, a partir de la información de consumo anual relevada y de la implementación de los cálculos de costo y actualización por inflación, es posible obtener para cada AH la media de consumo anual (kWh/año) y costo anual comparable (AR\$/año) para 2014 y 2018. Estos resultados por $\mathrm{AH}$, a su vez, pueden ser complementados con información censal y de consumo desagregado por usos, correspondientes al año 2014, la cual fue relevada de trabajos de investigación previos (Chévez, 2018; Chévez, Martini y Discoli, 2019a; 2019b). Esto amplía las posibilidades de análisis e inferencias (Tabla 3).

6. Se adoptó la información del Índice de Precios al Consumidor de la Ciudad Autónoma de Buenos Aires ya que éste cuenta con continuidad tanto en la publicación de los resultados como en el sostenimiento de su metodología durante el período bajo análisis. Por su parte, el Instituto Nacional de Estadísticas y Censos ha sido centro de debate respecto a la metodología empleada para medir este índice, además de presentar severas discontinuidades en la publicación de sus resultados. 
Tabla 3. Información socio-demográfica y energética asociada a cada una de las AH

\begin{tabular}{|c|c|}
\hline \multicolumn{2}{|l|}{ Variable/Indicador } \\
\hline \multicolumn{2}{|l|}{ Personas/hogar } \\
\hline \multicolumn{2}{|l|}{ Habitaciones/hogar } \\
\hline \multicolumn{2}{|l|}{ Necesidades Básicas Insatisfechas -NBI- [\%] ${ }^{7}$} \\
\hline \multicolumn{2}{|l|}{ Gas por red [\%] } \\
\hline \multirow[t]{3}{*}{ Tipología [\%] } & Casa \\
\hline & Departamento \\
\hline & Vivienda precaria \\
\hline \multirow[t]{3}{*}{ Calidad Constructiva [\%] } & Satisfactoria \\
\hline & Básica \\
\hline & Insuficiente \\
\hline \multirow{9}{*}{$\begin{array}{l}\text { Participación de los usos finales sobre el total de la demanda } \\
\text { eléctrica de cada } \mathrm{AH}[\%]\end{array}$} & Climatización (F/C) \\
\hline & Cocción \\
\hline & Agua Caliente Sanitaria (ACS) \\
\hline & Ref. de alimentos \\
\hline & Iluminación \\
\hline & Lavado de ropa \\
\hline & Informática y cel. \\
\hline & TV, audio y video \\
\hline & Otros electrodomésticos \\
\hline
\end{tabular}

Fuente: elaboración propia.

\subsection{Integración de la información: nivel de análisis global-urbano}

Un vez relevada y procesada la información en el nivel de análisis de las áreas homogéneas, es posible integrar la información a nivel global y obtener los resultados agregados de consumo anual (kWh/año) y costo anual comparable (AR\$/año) para 2014 y 2018. En relación a este aspecto, es preciso considerar que para la integración de los valores a escala urbana, es necesario calcular promedios ponderados que contemplen el peso de cada una de las áreas homogéneas. En consecuencia, para calcular el consumo (kWh/año) y el costo (AR\$/año) de la electricidad en toda la ciudad se aplica la Ecuación 2.

$$
\bar{X}_{v}=\sum_{i=1}^{n}\left[V_{i} *\left(\frac{\operatorname{Hog}_{i}}{\operatorname{Hog}_{\text {tot }}}\right)\right] \quad \text { Ecuación 2 }
$$

Dónde:

$\bar{X}_{v}=$ promedio ponderado de la variable $\mathrm{V}$ en la ciudad

$V_{i}=$ valor de la variable en la i-ésima área homogénea

$H g_{i}=$ cantidad de hogares en la i-ésima área homogénea

$\operatorname{Hog}_{\text {tot }}=$ cantidad de hogares totales en la ciudad

7. El nivel de NBI es un indicador de pobreza estructural, con lo cual permite la comparación transversal en el tiempo. 


\subsection{Comparativa de los resultados a nivel intra-urbano y global-urbano}

Una vez obtenidos los valores promedio por usuario de consumo eléctrico y costo anual del servicio para 2014 y 2018, desagregado por AH y para toda la ciudad, es posible establecer análisis pormenorizados sobre las variaciones ocurridas a partir de la RTI del año 2016. En efecto, será posible identificar si el valor global-urbano de la variación de consumo eléctrico es representativo para caracterizar los impactos de los cambios tarifarios en la ciudad o, si por el contrario, el análisis intra-urbano aporta información más precisa que amerita su desarrollo.

\section{Resultados del análisis temporal del consumo eléctrico intra-urbano y global-urbano (2014-2018)}

A partir de la utilización de la metodología planteada se relevaron los 278 casos (Figura 3) y se obtuvieron los resultados para el caso de estudio, los cuales se presentan a continuación. En primer lugar, se exponen los resultados desagregados -por áreas homogéneas-; luego, los resultados globales - para la ciudad completa- $y$, por último, la comparativa entre ambas escalas de trabajo.

\subsection{Resultados por área homogénea -análisis intra-urbano-: la importancia de identificar diferencias territoriales}

A partir del enfoque territorial intra-urbano es posible identificar comportamientos desiguales entre los diferentes sectores característicos de la ciudad. A continuación, en la Tabla 4 se pueden identificar los consumos y los costos reales anuales (2014 y 2018) de las cinco áreas homogéneas utilizadas para el análisis:

Tabla 4. Resultados por AH del consumo y costo real promedio por usuario en 2014 y 2018.

\begin{tabular}{|c|c|c|c|c|}
\hline \multicolumn{2}{|c|}{ Indicador } & Valor 2014 & Valor 2018 & Dif. Porcentual \\
\hline \multirow[t]{2}{*}{$\mathrm{AH} 1$} & kWh/año & 2187,87 kWh/año & 2162,72 kWh/año & $-1,15 \%$ \\
\hline & $\mathrm{AR} \$_{2018} / \mathrm{año}$ & 584,70 AR $\$_{2018}$ /año & 6452,55 AR $\$_{2018} /$ año & $+1104 \%$ \\
\hline \multirow[t]{2}{*}{$\mathrm{AH} 2$} & kWh/año & 2423,36 kWh/año & 2468,55 kWh/año & $+1,86 \%$ \\
\hline & $\mathrm{AR} \$_{2018} / \mathrm{año}$ & $664,12 \mathrm{AR}_{2018} /$ año & $7243,47 \mathrm{AR} \$_{2018} /$ año & $+1091 \%$ \\
\hline \multirow[t]{2}{*}{$\mathrm{AH} 3$} & kWh/año & 3370,10 kWh/año & 2937,30 kWh/año & $-12,84 \%$ \\
\hline & $\mathrm{AR} \$_{2018} / \mathrm{año}$ & $1083,06 \mathrm{AR}_{2018}$ /año & 8445,79 AR $\$_{2018} /$ año & $+780 \%$ \\
\hline \multirow[t]{2}{*}{$\mathrm{AH} 4$} & kWh/año & 3748,06 kWh/año & 4404,36 kWh/año & $+17,51 \%$ \\
\hline & $\mathrm{AR} \$_{2018} / \mathrm{año}$ & $1404,92 \mathrm{AR} \$_{2018} /$ año & $14618,42 \mathrm{AR} \$_{2018} /$ año & $+1041 \%$ \\
\hline \multirow[t]{2}{*}{$\mathrm{AH} 5$} & kWh/año & 3241,92 kWh/año & 3038,14 kWh/año & $-6,29 \%$ \\
\hline & $\mathrm{AR} \$_{2018} / \mathrm{año}$ & $1114,76 \mathrm{AR}_{2018} /$ año & $8306,58 \mathrm{AR} \$_{2018} /$ año & $+745 \%$ \\
\hline
\end{tabular}

Fuente: elaboración propia.

En este sentido, se observa que en términos de consumo eléctrico el $\mathrm{AH} 1$ presentó una leve caída (-1,15\%); el AH2 un leve crecimiento (+1,86\%); el AH3 tuvo una fuerte caída en su demanda $(-12,84 \%)$; el AH4 creció de una manera muy importante $(+17,51 \%)$; mientras que el AH5 presentó una caída considerable (-6,29\%). En este punto ya es posible señalar que un valor global para toda la ciudad, el cual se presentará en la siguiente sección, no permitiría visualizar situaciones muy diversas a lo largo del territorio donde algunas áreas se han mantenido relativamente estables ( $\mathrm{AH} 1$ y $\mathrm{AH} 2$ ); otras han crecido significativamente (AH4); mientras que otras 
han sufrido una fuerte caída (AH3 y AH5). Esto significa que si se utilizara el valor global-urbano como insumo para el diseño de políticas, su efectividad se vería limitada dado que el territorio demuestra una importante heterogeneidad.

En relación a este último aspecto, a continuación se analizan variables asociadas para cada una de las áreas homogéneas (Tabla 5), las cuales nos permiten realizar inferencias acerca de los cambios detectados en la media de consumo eléctrico por usuario entre 2014 y 2018. Las mismas incluyen información socio-demográfica extraída del Censo 2010; como así también la participación porcentual de nueve usos finales dentro de la demanda eléctrica relevados por medio de encuestas. Dicha información fue extraída de Chévez (2018) y Chévez et al. (2019a).

Tabla 5. Información socio-demográfica y energética asociada a cada una de las AH

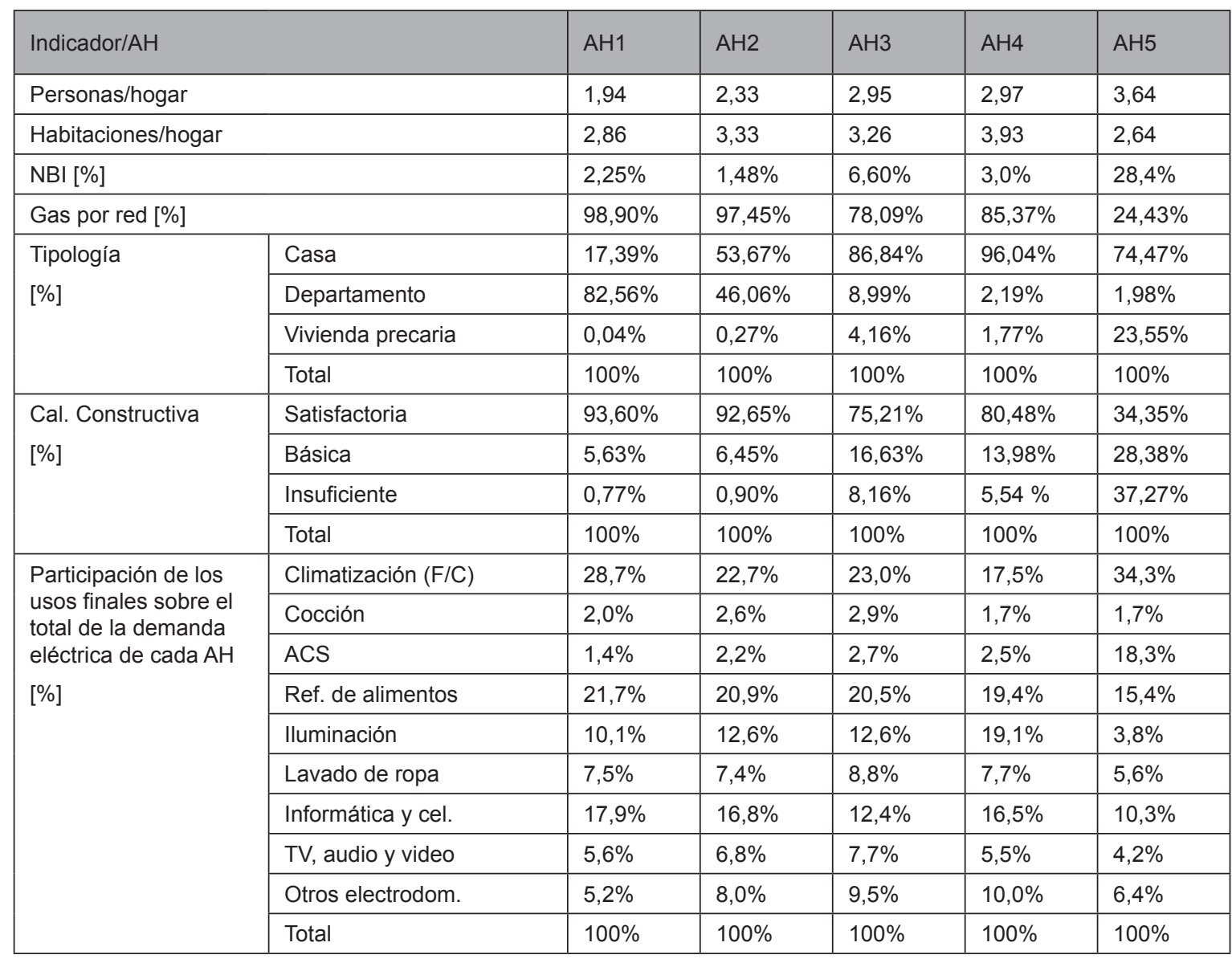

Fuente: Chévez (2018) y Chévez et al. (2019a)

A partir de las variables relevadas para cada una de las áreas homogéneas, es posible establecer consideraciones acerca de los motivos e implicancias de los cambios en el consumo eléctrico en el intervalo analizado.

Por ejemplo, en el AH1, ubicada en el centro del casco fundacional, la demanda por usuario se redujo levemente $(-1,15 \%)$, a pesar de que el pago anual por la energía fue el que sufrió mayores incrementos en relación a las cinco áreas $(+1104 \%)$ y alcanzó un valor de $6452,55 \mathrm{AR} \$_{2018} /$ año. En este sentido, es posible verificar que los niveles de NBI en esta AH son bajos (2,25\%), lo cual indica que en promedio la población prácticamente ha podido sostener sus niveles de consumo energético puesto que cuentan con capacidad de pago, pero que sin embargo al incrementarse en 
unas once veces el mismo, su incidencia sobre el nivel de ingreso es mucho mayor y por consiguiente son erogaciones que no se realizan en otro tipo de gastos. Por otro lado, esta AH cuenta con una amplia cobertura de gas por red (98,9\%), las viviendas son mayormente departamentos $(82,56 \%)$ y la calidad constructiva es mayoritariamente satisfactoria $(93,6 \%)$, con una media de 1,94 personas/hogar. Esto se traduce en viviendas compactas, con bajos coeficientes volumétricos de pérdida de calor por medio de la envolvente, las cuales, además, principalmente resuelven los usos térmicos por medio de la red de gas. Asimismo, el AH1 presenta la menor demanda anual por vivienda de las cinco áreas homogéneas: 2162,72 kWh/año (2018).

El AH2, que rodea el AH1 y alcanza los límites del casco urbano fundacional, presentó un comportamiento prácticamente estable, incrementando su demanda por usuario en un $+1,86 \%$, a pesar de que el incremento en el pago anual por la energía se incrementó en más de diez veces $(+1091 \%)$ y alcanzó un valor de $7243,47 \mathrm{AR}{ }_{2018} / \mathrm{año}$. El análisis del nivel de NBI en esta área $(1,48 \%)$ permite identificar que se trata de un sector con poca población con niveles de precariedad habitacional, lo cual ha permitido a su población sostener e incluso incrementar levemente su consumo, abonando el servicio a un costo mucho mayor que en el período previo. Por ende, este re-direccionamiento de los ingresos probablemente haya ido en detrimento de otro tipo de gastos familiares. En tanto, esta AH presenta condiciones similares al AH1 en cuanto a la cobertura de gas por red $(97,45 \%)$ y proporción de viviendas con calidad constructiva satisfactoria $(92,65 \%)$, sin embargo las tipologías de viviendas en este caso se reparten prácticamente en partes iguales entre casas y departamentos $(53,67 \%$ y $46,06 \%$ respectivamente), con una media de personas por hogar de 2,33. Las mencionadas condiciones de compacidad, consolidación y calidad constructiva en el sector favorecen la disminución de las pérdidas térmicas. En consecuencia, la demanda de electricidad resulta levemente superior al del AH1, alcanzando una media por hogar de 2468,55 kWh/año (2018).

El AH3, que se extiende desde los límites del casco fundacional hasta intersectarse con el periurbano, presenta una situación más comprometida dado que su consumo cayó significativamente $(-12,84 \%)$. En este sentido, el incremento porcentual del costo de la energía fue uno de los menos elevados $(+780 \%)$, pero en términos absolutos es el sector con el segundo valor más alto $(8445,79$ $\mathrm{AR} \$_{2018} / \mathrm{año}$ ). Además, es preciso considerar que en el caso de haber sostenido sus niveles de consumo, el incremento del costo hubiera sido entre nueve y diez veces respecto de 2014. El caso particular del AH3 indica que un 6,60\% de la población cuenta con algún tipo de necesidad básica insatisfecha (NBI) y la proporción de viviendas con calidad satisfactoria únicamente es del 75,21\%; denotando que las condiciones de precariedad son mayores en relación a las áreas AH1 y AH2. Por su parte, es preciso señalar que la cobertura de gas por red del AH3 es del 78,09\%, por lo tanto aproximadamente un quinto de la población depende exclusivamente de la electricidad o el gas licuado de petróleo (GLP) para satisfacer usos energo-intensivos como calefacción, cocción y agua caliente sanitaria. Esto significa que la reducción de la demanda eléctrica en sectores con baja y media cobertura de gas por red puede estar impactando en usos finales de extrema importancia, como es el caso de la calefacción, que permite mantener niveles de confort interior en las viviendas. En efecto, este sector urbano, no ha podido sostener sus niveles de consumo en relación a la etapa previa. En este caso, las tipologías de vivienda son mayormente casas (86,84\%) y la media de personas por hogar es de 2,95; y su demanda anual para 2018 decayó a 2937,30 kWh/año.

El AH4, ubicada en el eje noroeste del partido, se trata de un sector con segmentos poblacionales de alto poder adquisitivo y presenta un comportamiento diametralmente opuesto a los restantes 
sectores analizados, puesto que su consumo se ha incrementado en un $+17,51 \%$. Allí, el monto promedio anual destinado al pago de la electricidad se incrementó de forma considerable $(+1041 \%)$ y alcanzó un valor absoluto promedio de $14.618,42 \mathrm{AR} \$_{2018} / \mathrm{año}$, que es el más alto de las cinco áreas estudiadas. Si bien se trata de un sector urbano donde están emplazados barrios que tradicionalmente cuentan con altos niveles de ingresos, como así también barrios cerrados o countries, el área cuenta con un nivel de NBI del 3\% y la proporción de viviendas con calidad constructiva satisfactoria es del $80,48 \%$. Esto indica que los mencionados barrios coexisten con una cierta proporción de viviendas con condiciones de precariedad habitacional. Por otro lado, la cobertura del gas por red es del 85,37\%, la tipología de vivienda predominante son las casas $(96,04 \%)$ y la media de personas por hogar es de 2,97. El AH4 cuenta con una media de personas por hogar similar al del AH3 y una cobertura de gas por red superior, lo cual se debería corresponder en un consumo eléctrico inferior. Sin embargo, el consumo registrado es notoriamente mayor (4404,36 kWh/año), excediendo el del AH3 en $+49 \%$. Esto se debe fundamentalmente al equipamiento detectado y al tamaño de las viviendas. Cabe señalar que esta diferencia en 2014 era de $+11 \%$, sin embargo ambos sectores presentaron comportamientos opuestos, uno en declive y el otro en alza. Finalmente, es necesario esbozar una posible explicación respecto del incremento del consumo en esta $\mathrm{AH}$. En este sentido, probablemente, las viviendas continuaron con la incorporación de equipamiento domiciliario, fundamentalmente aquellas que aún no habían alcanzado la saturación en su parque de equipos. En consecuencia, se infiere que la suba en el consumo energético está relacionada a la inserción de nuevos electrodomésticos y no a una mayor cantidad de horas de uso de los ya existentes.

El AH5 presenta, en su mayor parte, radios censales de consolidación baja y su posición en el territorio es periférica, es decir que ocupa los bordes del trazado urbano. Como condición principal, es necesario destacar que el nivel de NBI es del 28,4\%. La variación en la demanda eléctrica fue de -6,29\%. Si bien no es el AH que mayor reducción presentó; un descenso como el detectado, en un sector donde la cobertura de gas natural por red es muy limitada $(24,43 \%)$, significa que algunos usos de vital importancia como la calefacción, cocción o agua caliente sanitaria, que en otras AHs son satisfechos por gas natural, han tenido que ser reducidos en su utilización. En consecuencia, con dicha reducción se infiere que los niveles de confort han descendido. Tal como se muestra en la Tabla 5, los usos de climatización y agua caliente sanitaria en el AH5 son muy superiores a los de las restantes AHs (34,3\% y 18,3\% respectivamente), ratificando las afirmaciones previas. Asimismo, su consumo promedio es cercano al del AH3 (ambos cercanos a los 3000 $\mathrm{kWh} / \mathrm{año}$ ), pero con una media de personas por hogar y de cobertura de gas por red muy diferente: $\mathrm{AH} 3=2,95$ y $\mathrm{AH} 5=3,64$ personas por hogar y $\mathrm{AH} 3=78,09 \%$ y $\mathrm{AH} 5=24,43 \%$ de cobertura. Estos valores indican que el consumo del AH5 debería ser superior, puesto que debe satisfacer usos que el AH3 satisface con gas natural por red y además presenta más personas en las viviendas. Para complementar la información, se identifica que un 74,47\% de las viviendas son casas y un $23,55 \%$ son viviendas precarias; por su parte, sólo un $34,35 \%$ de las viviendas presenta una calidad constructiva satisfactoria.

En consecuencia, sintetizando el análisis es posible afirmar que: (i) En los sectores de mayor consolidación urbana (AH1 y AH2), los cuales presentan alta densidad habitacional (personas/ha.), baja cantidad de personas por hogar, predominancia de viviendas con tipología de departamento, acceso prácticamente total a la red de gas natural y bajos niveles de NBI, la demanda eléctrica ha permanecido estable entre 2014-2018. Allí, los montos de las facturas resultan los menores de la ciudad, dado que corresponden a consumos reducidos y los usos térmicos (de mayor incidencia en la demanda) son resueltos fundamentalmente por el gas natural. (ii) En el sector de mayor po- 
der adquisitivo de la ciudad (AH4), el cual es un área de baja consolidación, la demanda eléctrica ha crecido significativamente $(+17,51 \%)$, infiriendo que las viviendas de este sector han continuado incorporando equipamiento domiciliario y, por tal motivo, el promedio por usuario creció notablemente. (iii) Por último, en los sectores de baja consolidación y altos niveles de NBI (AH3 y AH5), donde la cobertura de gas por red es acotada, la demanda eléctrica cayó notablemente, lo cual representa un grave problema dado que buena parte de la energía eléctrica es destinada a satisfacer usos térmicos, los cuales en otras áreas son satisfechos mediante el gas natural. Por lo tanto, existe una alta probabilidad de que estas viviendas hayan reducido sus niveles de confort interior, con un consecuente deterioro de su calidad de vida.

\subsection{Resultados globales para la ciudad de La Plata-análisis global-urbano-: la generalidad de los datos agregados}

Aplicando la Ecuación 2 presentada en la sección metodológica, que pondera el peso y el resultado de cada AH sobre la ciudad, se obtuvo la variación del consumo y del costo promedio anual entre 2014 y 2018 del caso de estudio (Tabla 6). Allí se observa que a nivel general la demanda anual de energía eléctrica varió en -3,33\%, pasando de 2924 a $2829 \mathrm{kWh} /$ año; y que su costo real se incrementó aproximadamente nueve veces (+916\%), pasando de 915,13 a 8380,82 AR \$ 2018 /año (costo por la energía eléctrica sin impuestos).

Tabla 6. Resultados agregados a nivel ciudad del consumo y costo promedio por usuario en 2014 y 2018.

\begin{tabular}{|l|l|l|l|}
\hline Indicador & Valor 2014 & Valor 2018 & Dif. porcentual \\
\hline kWh/año & $2924,20 \mathrm{kWh} / \mathrm{año}$ & $2829,83 \mathrm{kWh} / \mathrm{año}$ & $-3,33 \%$ \\
\hline $\mathrm{AR} \$_{2018} / \mathrm{año}$ & $915,13 \mathrm{AR} \$_{2018} /$ año & $8380,82 \mathrm{AR} \$_{2018} / \mathrm{año}$ & $+916 \%$ \\
\hline
\end{tabular}

Fuente: elaboración propia.

En este punto resulta de interés analizar las diferencias que se detectan entre los resultados individuales de las áreas homogéneas y el resultado global de toda el área de estudio, a los efectos de resaltar la importancia de emprender estudios territoriales detallados.

\subsection{Comparativa entre los niveles de análisis}

Una lectura inicial de una reducción en el consumo energético global del -3,33\%, que no contara con los datos desagregados territorialmente, podría sostener que el incremento del costo de las facturas eléctricas en prácticamente nueve veces produjo cambios leves en los comportamientos energéticos de los usuarios de la ciudad de La Plata. Sin embargo, tal como se identificó en el análisis desagregado, hubo sectores que llegaron a reducir su demanda en un -12,84\% y otros que lo han incrementado en un $+17,51 \%$; por lo que el análisis global presentaría un sesgo importante en sus resultados. A partir de la identificación de los mencionados valores es notorio que los análisis globales pueden brindar una aproximación inicial al objeto de estudio; sin embargo, es necesario abordar niveles inferiores de desagregación, que pueden ser territoriales, socio-demográficos, etc., que permitan identificar las particularidades del sector residencial. Esta diferenciación, en última instancia, permitirá direccionar políticas específicas para cada sector, las cuales deberán corresponderse con las necesidades particulares de cada uno de éstos. 
Asimismo, es preciso destacar que el mercado laboral en la región de estudio ha incrementado su nivel de desocupación entre 2014 y 2018 , pasando de 7,2\% a 8,3\%. Esta variable, en consonancia con los importantes incrementos tarifarios, puede explicar la caída global del consumo eléctrico residencial en el caso de estudio (-3,33\%). En tanto, es probable que el indicador de desocupación también haya sido heterogéneo en el territorio. En esa dirección, es probable que el indicador haya crecido de forma más notoria en las áreas con mayor nivel de NBI, donde se presentan situaciones de mayor precariedad e informalidad laboral. Este hecho presenta un estrecho vínculo con los resultados desagregados obtenidos en el presente trabajo.

Por su parte, continuando con el análisis de los resultados, es posible plantear como hipótesis que ante una tarifa accesible como la del año 2014 los usos energéticos no tendían al derroche, sino al de la satisfacción de los requerimientos necesarios de las viviendas; el cual, hacia 2018 luego de la RTI, algunos usuarios optaron por sostener en desmedro de la adquisición de otros bienes de consumo, mientras que otros no lo pudieron hacer y solo una reducida proporción logró incrementarlo.

Para validar la mencionada hipótesis y establecer nuevas inferencias sería necesario desarrollar encuestas detalladas que indaguen sobre cambios de hábitos en el uso de equipamientos clave y analicen la cobertura de las necesidades energéticas mínimas necesarias; lo cual se plantea como continuidad del presente trabajo. Estos resultados serán de utilidad tanto por área homogénea como a nivel global.

\section{Conclusiones}

A partir del desarrollo y la implementación de la metodología propuesta fue posible verificar la hipótesis planteada al comienzo del trabajo. En este sentido, podemos afirmar que es de vital importancia incentivar y promover la ejecución de estudios energéticos con anclaje territorial desagregados, dado que éstos permiten profundizar la comprensión de estudios globales (de información agregada), interpelando sus resultados y proveyendo información más precisa que puede ser de utilidad para el diseño preciso de políticas públicas.

En consecuencia, los resultados obtenidos permitieron comprender de forma precisa el impacto de la RTI en el territorio. En este sentido, la inserción de variables socio-demográficas y energéticas contribuyó al análisis de los resultados obtenidos, puesto que aspectos tales como los niveles de consolidación urbana, el NBI, el equipamiento predominante, etc. dieron indicios para inferir los motivos de los incrementos o decrecimientos en el consumo de energía residencial.

En relación a este aspecto, los resultados del caso de estudio (ciudad de La Plata) entre 2014 y 2018 demostraron diferencias sustanciales entre el valor obtenido para toda la ciudad y para cada una de las áreas homogéneas. En efecto, si únicamente se tuviera en cuenta el resultado global, podría afirmarse que los efectos del incremento de las tarifas energéticas y la caída en la actividad del mercado laboral han provocado un leve decrecimiento en los usuarios platenses $(-3,33 \%)$, lo cual es válido pero incompleto. En consecuencia, dicho análisis dejaría de lado la identificación de sectores de la ciudad donde las consecuencias fueron más pronunciadas, como el AH3 $(-12,84 \%)$ y el AH5 (-6,29\%); sectores donde el consumo continuó su tendencia de crecimiento, como el AH4 (+17,51\%); o sectores que han variado levemente su demanda eléctrica, como el AH1 (-1,15\%) y el AH2 (+1,86\%). 
A modo de síntesis, el análisis pormenorizado de las variables socio-demográficas y energéticas de las cinco áreas homogéneas permitió identificar algunas directrices generales. En este sentido, se observa que en los sectores de mayor consolidación urbana y bajos niveles de NBI la demanda eléctrica ha permanecido estable entre 2014-2018. En el sector de mayor poder adquisitivo de la ciudad, el cual presenta una baja consolidación y bajo nivel de NBI, la demanda eléctrica ha crecido significativamente, infiriendo que las viviendas de este sector han continuado incorporando equipamiento domiciliario y, por tal motivo, el promedio de consumo por usuario creció notablemente. Por último, en los sectores de baja consolidación, altos niveles de NBI y escasa cobertura de gas por red, la demanda eléctrica cayó notablemente, con la probabilidad de que estas viviendas hayan reducido sus niveles de confort interior $y$, consecuentemente, su calidad de vida. En este sentido, es probable que en estas áreas se ubiquen numerosos beneficiarios de la denominada 'tarifa social', la cual está dirigida a la población económicamente más vulnerable, bonifica una parte de sus consumos y otorga una tarifa a un precio más bajo que la de los restantes usuarios. En efecto, dados los resultados obtenidos, y en consonancia con otros estudios abordados por los autores (Chévez, San Juan y Martini, 2019), es necesario señalar que la 'tarifa social' ha sido insuficiente para cubrir la atención de las necesidades básicas de sus beneficiarios, el cual era su objetivo principal. Esto es notorio ya que la demanda en los sectores con alto NBI cayó notablemente.

Por lo señalado previamente resulta imposible establecer una conclusión única respecto del comportamiento del consumo eléctrico ante el incremento tarifario impulsado por la RTI de 2016. En efecto, ante un aumento de las facturas similar en las cinco AH (entre siete y once veces), cada una de éstas reaccionó de manera diferenciada. Dos de éstas mantuvieron su demanda (AH1 y $\mathrm{AH} 2)$, dos la disminuyeron (AH3 y AH5) y una la incrementó considerablemente (AH4).

Asimismo, es posible inferir que en los sectores donde la demanda bajó notablemente ante el incremento del costo (AH3 y AH5) las reducciones no se dieron por una mejora en la eficiencia, que sería lo deseable; sino que, por los altos niveles de NBI, probablemente dichos sectores hayan sufrido las peores consecuencias de la caída del mercado laboral, hayan tenido que recortar sus horas de uso en consumos vitales como la calefacción para poder afrontar los pagos del servicio. En dicho contexto, es difícil sostener que en un sector donde los niveles de NBI alcanzan el 6,6\% y $28,4 \%$ de la población, los usuarios hayan invertido en mejorar la aislación térmica de sus viviendas o realizar el recambio de sus equipos energo-intensivos.

A modo de cierre, es preciso señalar que cualquier política energética que vaya a tener incidencia sobre los patrones de consumo de los usuarios va a repercutir sobre la calidad de vida de éstos. Por lo tanto, necesariamente se deberán tomar en consideración las diferencias y particularidades de los sectores que conforman la totalidad del territorio, analizar las variables e indicadores que los definen y formular estrategias que sean concordantes con las diversas realidades sociales y tecnológicas en el territorio. De esta forma, el diseño de las políticas públicas podría ser más preciso y, así, repercutirán más equitativamente sobre la población. 


\section{Anexo 1}

Tabla A1. Cuadros tarifarios de la distribuidora local para el año 2014 y 2018 (enero-febrero y marzodiciembre). Tarifa regular en pesos argentinos sin impuestos.

\begin{tabular}{|c|c|c|c|c|c|c|c|c|}
\hline \multicolumn{3}{|l|}{2014} & \multicolumn{6}{|l|}{2018} \\
\hline \multicolumn{3}{|c|}{ Cuadro tarifario 2014} & \multicolumn{3}{|c|}{ Cuadro tarifario enero-febrero 2018} & \multicolumn{3}{|c|}{ Cuadro tarifario marzo-diciembre 2018} \\
\hline \multicolumn{3}{|c|}{ Tarifa regular } & \multicolumn{3}{|c|}{ Tarifa regular } & \multicolumn{3}{|c|}{ Tarifa regular } \\
\hline $\begin{array}{l}\text { Rango } \\
\text { [kWh/ } \\
\text { bim] }\end{array}$ & $\begin{array}{l}\text { Cargo Fijo } \\
{[\mathrm{AR} \$ / \mathrm{bim}]}\end{array}$ & $\begin{array}{l}\text { Cargo } \\
\text { variable } \\
\text { [AR\$/ kWh] }\end{array}$ & $\begin{array}{l}\text { Rango } \\
\text { [kWh/ } \\
\text { mes] }\end{array}$ & $\begin{array}{l}\text { Cargo Fijo } \\
{[A R \$ / \text { mes] }}\end{array}$ & $\begin{array}{l}\text { Cargo } \\
\text { variable } \\
\text { [AR\$/ kWh] }\end{array}$ & $\begin{array}{l}\text { Rango } \\
\text { [kWh/mes] }\end{array}$ & $\begin{array}{l}\text { Cargo Fijo } \\
\text { [AR } \$ / \text { mes] }\end{array}$ & $\begin{array}{l}\text { Cargo } \\
\text { variable } \\
\text { [AR\$/ kWh] }\end{array}$ \\
\hline $0-300$ & 5,96 & 0,107 & $0-150$ & 51,30 & 1,928 & $0-150$ & 61,28 & 2,549 \\
\hline $301-650$ & 24,71 & 0,058 & $151-325$ & 126,19 & 1,773 & $151-325$ & 153,24 & 2,367 \\
\hline $651-800$ & 24,71 & 0,066 & $326-400$ & 163,43 & 1,794 & $326-400$ & 196,68 & 2,392 \\
\hline $801-900$ & 34,41 & 0,070 & $401-450$ & 229,05 & 1,869 & $401-450$ & 273,23 & 2,480 \\
\hline $901-1000$ & 41,22 & 0,079 & $451-500$ & 276,80 & 1,911 & $451-500$ & 328,93 & 2,528 \\
\hline $1001-1200$ & 56,66 & 0,171 & $501-600$ & 475,03 & 2,082 & $501-600$ & 560,16 & 2,728 \\
\hline $1201-1400$ & 66,56 & 0,192 & $601-700$ & 658,49 & 2,212 & $601-700$ & 774,17 & 2,880 \\
\hline $1401-2800$ & 74,76 & 0,293 & $701-1400$ & 955,62 & 2,292 & $701-1400$ & 1120,77 & 2,974 \\
\hline$>2800$ & 75,32 & 0,471 & $>1400$ & 1149,69 & 2,309 & $>1400$ & 1347,15 & 2,993 \\
\hline
\end{tabular}

Fuente: OCEBA (2019).

Tabla A2. Cuadros tarifarios de la distribuidora local para el año 2014 y 2018 (enero-febrero y marzodiciembre). Tarifa social en pesos argentinos sin impuestos.

\begin{tabular}{|c|c|c|c|c|c|c|c|c|}
\hline \multirow{2}{*}{\multicolumn{3}{|c|}{$\begin{array}{l}2014 \\
\text { Tarifa Social }\end{array}$}} & \multicolumn{6}{|l|}{2018} \\
\hline & & & \multicolumn{3}{|l|}{ Tarifa Social } & \multicolumn{3}{|l|}{ Tarifa Social } \\
\hline $\begin{array}{l}\text { Rango } \\
\text { [kWh/bim] }\end{array}$ & $\begin{array}{l}\text { Cargo Fijo } \\
\text { [AR\$/ bim] }\end{array}$ & $\begin{array}{l}\text { Cargo } \\
\text { variable } \\
\text { [AR\$/ kWh] }\end{array}$ & $\begin{array}{l}\text { Rango } \\
\text { [kWh/mes] }\end{array}$ & $\begin{array}{l}\text { Cargo Fijo } \\
{[A R \$ / \text { mes] }}\end{array}$ & $\begin{array}{l}\text { Cargo } \\
\text { variable } \\
\text { [AR\$/ kWh] }\end{array}$ & $\begin{array}{l}\text { Rango } \\
{[\mathrm{kWh} / \mathrm{mes}]}\end{array}$ & $\begin{array}{l}\text { Cargo Fijo } \\
\text { [AR } \$ / \text { mes] }\end{array}$ & $\begin{array}{l}\text { Cargo } \\
\text { variable } \\
{[A R \$ / k W h]}\end{array}$ \\
\hline $0-300$ & $\mathrm{~N} / \mathrm{A}$ & $\mathrm{N} / \mathrm{A}$ & $0-150$ & 51,3 & 0 & $0-150$ & 61,28 & 0 \\
\hline $301-650$ & $\mathrm{~N} / \mathrm{A}$ & $\mathrm{N} / \mathrm{A}$ & $151-300$ & 126,19 & 1,112 & $151-300$ & 153,24 & 1,510 \\
\hline $651-800$ & $\mathrm{~N} / \mathrm{A}$ & $\mathrm{N} / \mathrm{A}$ & $301-325$ & 126,19 & 1,773 & $301-325$ & 153,24 & 2,367 \\
\hline $801-900$ & $\mathrm{~N} / \mathrm{A}$ & $\mathrm{N} / \mathrm{A}$ & $326-400$ & 163,43 & 1,794 & $326-400$ & 196,68 & 2,392 \\
\hline $901-1000$ & $\mathrm{~N} / \mathrm{A}$ & $\mathrm{N} / \mathrm{A}$ & $401-450$ & 229,05 & 1,869 & $401-450$ & 273,23 & 2,480 \\
\hline $1001-1200$ & $\mathrm{~N} / \mathrm{A}$ & $\mathrm{N} / \mathrm{A}$ & $451-500$ & 276,8 & 1,911 & $451-500$ & 328,93 & 2,528 \\
\hline $1201-1400$ & $\mathrm{~N} / \mathrm{A}$ & $\mathrm{N} / \mathrm{A}$ & $501-600$ & 475,03 & 2,082 & $501-600$ & 560,16 & 2,728 \\
\hline $1401-2800$ & $\mathrm{~N} / \mathrm{A}$ & $\mathrm{N} / \mathrm{A}$ & $601-700$ & 658,49 & 2,212 & $601-700$ & 774,17 & 2,880 \\
\hline$>2800$ & $\mathrm{~N} / \mathrm{A}$ & $\mathrm{N} / \mathrm{A}$ & $701-1400$ & 955,62 & 2,292 & $701-1400$ & 1120,77 & 2,974 \\
\hline- & - & - & $>1400$ & 1149,69 & 2,309 & $>1400$ & 1347,15 & 2,993 \\
\hline
\end{tabular}

Fuente: OCEBA (2019). 


\section{Bibliografía}

- Bondorevsky, D. (2016). Un haz de luz sobre la Revisión Tarifaria Integral en Distribución Eléctrica en el AMBA. Recuperado de: <https://www.cippec.org/publicacion/un-haz-de-luz-sobre-la-revision-tarifaria-integral-en-distribucion-electrica-en-el-amba/>

- Cammesa (2019). Datos e Informes. Datos Sintesis Mensual. Recuperado de: <https://portalweb.cammesa.com/ memnet1/Pages/descargas.aspx>

- Chévez, P. (2018). Construcción de escenarios urbano-energéticos a partir de la implementación de estrategias de eficiencia energética y energías renovables en el sector residencial (Tesis de doctorado). Universidad Nacional de Salta: Salta.

- Chévez, P., Discoli, C. y Martini, I. (2017). El consumo de gas por red en el sector residencial. Análisis territorial y temporal. Realidad Económica, (308), 117-137.

- Chévez, P., Martini, I. y Discoli, C. (2018). Análisis territorial y temporal del consumo eléctrico en el sector residencial de Argentina (1995-2014). Cuadernos Geográficos de la Universidad de Granada, (57), n², 162-188. doi: $<$ http://dx.doi.org/10.30827/cuadgeo.v57i2.5923>

- Chévez, P., Martini, I. y Discoli, C. (2019a). Methodology developed for the construction of an urban-energy diagnosis aimed to assess alternative scenarios: An intra-urban approach to foster cities' sustainability. Applied Energy, (237), pp. 751-778. doi: <https://doi.org/10.1016/j.apenergy.2019.01.037>

- Chévez, P., Martini, I. y Discoli, C. (2019b). Desarrollo metodológico para la construcción de escenarios urbanoenergéticos de largo plazo. Cuaderno Urbano, (26), pp. 69-108. doi: <https://doi.org/10.30972/crn.26263791>

- Chévez, P., San Juan, G. y Martini, I. (2019). Alcances y limitaciones de la 'tarifa social' eléctrica en urbanizaciones informales (La Plata, Buenos Aires). Estudios Socioterritoriales, (26), pp. 1-23. doi: https://doi.org/10.37838/ unicen/est.26-034

- DGEyC-CBA (2019). Temperatura media, máxima media y mínima media $\left({ }^{\circ} \mathrm{C}\right)$. Enero 1991 - abril 2019. Dirección General de Estadística y Censo de la Ciudad de Buenos Aires. Recuperado de: <https://www.estadisticaciudad. gob.ar/eyc/?p=27702>

- DGEyC-CBA (2020). IPCBA. Evolución del Nivel General, de los bienes y de los servicios. Índices y variaciones porcentuales respecto del mes anterior. Ciudad de Buenos Aires. Julio de 2012/mayo de 2020. Dirección General de Estadística y Censo de la Ciudad de Buenos Aires. Recuperado de: <https://www.estadisticaciudad.gob.ar/ eyc/?p=27386>

- Discoli, C. (2009). Metodología para el diagnóstico urbano-energético-ambiental en aglomeraciones intermedias. El caso del Gran La Plata. La Plata, Argentina: Editorial Universitaria de La Plata.

- Durán, R. (2018a). Evaluación de aspectos socio-culturales para la planificación regional e implementación de energías renovables en Salta (Tesis de doctorado). Universidad Nacional de Salta: Salta.

- Durán, R. (2018b). Apuntes sobre pobreza energética. Estimaciones para Argentina. Años 2003-2018. Recuperado de: < https://tallerecologista.org.ar/publicacion/apuntes-sobre-pobreza-energetica/>

- Fraschina, S. Coord. (2018a). Argentina es el país de la región de mayor incremento en el peso de los servicios públicos sobre los salarios, en los últimos tres años (UNdAV). Recuperado de: <https://gallery.mailchimp. com/219db79b15bd598643ad6537b/files/2ae88708-d64e-482c-a483-2ce06bf7def2/Infograf\%C3\%ADa_Costo_ Tarifario.pdf >

- Fraschina, S. Coord. (2018b). En los últimos tres años, los servicios públicos aumentaron hasta un 2.000\% (UNdAV). Recuperado de: <http://undav.edu.ar/general/recursos/adjuntos/22096.pdf>

- IDEHAB (2005). Actividades habilitantes para la Segunda Comunicación Nacional de la República Argentina a la Convención Marco de las Naciones Unidas sobre Cambio Climático. La Plata, Argentina: Instituto de Estudios del Hábitat.

- INDEC (2018a). Encuesta Nacional de Gastos de los Hogares 1996/1997. Buenos Aires: Instituto Nacional de Estadísticas y Censos.

- INDEC (2018c). Encuesta Nacional de Gastos de los Hogares 2012/2013. Buenos Aires: Instituto Nacional de Estadísticas y Censos.

- INDEC (2019). Proyecciones y estimaciones. Buenos Aires: Argentina: Instituto Nacional de Estadísticas y Censos. 
- INDEC (2020a). Trabajo e ingresos. Mercado de trabajo. Tasa de desocupación en Aglomerados Urbanos. Instituto Nacional de Estadísticas y Censos. Recuperado de: <https://sitioanterior.indec.gob.ar/series_historicas.asp?id_ tema_1=4\&id_tema_2=31\&id_tema_3=58>

- INDEC (2020b). Informes técnicos. Mercado de trabajo: principales indicadores. Instituto Nacional de Estadísticas y Censos. Recuperado de: <https://www.indec.gob.ar/indec/web/Institucional-Indec-InformesTecnicos-58>

- Infoleg (2019). Resolución 6/2016. CABA Argentina: Ministerio de Energía y Minería.

- Margulis, D. (2014). Análisis de los determinantes de la demanda residencial de energía eléctrica en Argentina (Tesis de maestría). Universidad de Buenos Aires: CABA.

- OCEBA (2019). Cuadros Tarifarios para Distribuidoras con Contrato de Concesión Municipal y Provincial. Organismo de Control de Energía Eléctrica de la Provincia de Buenos Aires. Recuperado de: <https://oceba.gba.gov. $\mathrm{ar} / \mathrm{web} / \mathrm{s} . \mathrm{php}$ ? $\mathrm{i}=17>$

- Rodríguez, L. (2016). Instrumentación metodológica para el reciclado masivo de la edilicia residencial orientada a la eficiencia energética (Tesis de doctorado). Universidad Nacional de Salta: Salta.

- Soijet, M.; Rodríguez, M.; Mantovani, G.; Tonini, R. y Peralta, M. (2016). Ciudades intermedias. Alcances de la denominación. Santa Fe-Paraná ¿En el inicio de la consolidación metropolitana? Arquisur (10), 26-38.

- Tu Tiempo (2019). Clima La Plata Aeródromo. Datos climáticos: 1973 - 2019. Recuperado de: <https://www.tutiempo.net/clima/ws-875930.html>

\section{Sobre los autores}

Dr. Pedro Chévez

Doctor en Ciencias y especialista en Energías Renovables por la Universidad Nacional de Salta (UNSa), Arquitecto por la Universidad Nacional de La Plata. Investigador en el IIPAC-CONICET-UNLP. Desarrolla su actividad en la definición, ensayo y evaluación de escenarios urbano-energéticos de largo plazo para el sector residencial; aborda la demanda y la oferta de energía en distintas escalas, con especial énfasis en el análisis del impacto de la inserción de medidas de eficiencia energética y energías renovables. Asimismo, se desempeña como Ayudante de Curso Diplomado en la Universidad Nacional de La Plata (UNLP), en la Facultad de Arquitectura y Urbanismo.

Dra. Irene Martini

Doctora en Ciencias, área Energías Renovables de la Universidad de Salta. Magister en Políticas Ambientales y Territoriales, en el Instituto de Geografía FILO-UBA. Arquitecta por la Universidad Nacional de La Plata. Investigadora Independiente del CONICET en el IIPAC-CONICET-UNLP. Desarrolla su actividad en investigación en los campos del hábitat, la energía y el ambiente. Cuenta con más de 80 artículos a congresos y en revistas con referato de la especialidad. Ha participado en más de 26 proyectos de investigación CONICET, ANPCyT y UNLP. Es ACD ordinario de la cátedra de Estructuras de la FAU-UNLP y DocenteInvestigadora Categoría II.

Dr. Carlos Discoli

Doctor en Ciencias de la Universidad Nacional de Salta, Área: Energías renovables. Máster en Ambiente y Patología Ambiental de la Universidad Nacional de La Plata y la Escuela de los Altos Estudios de Siena. Ingeniero Mecánico de la Universidad Tecnológica Nacional, Regional La Plata. Investigador Principal del CONICET en IIPAC-CONICET-UNLP. Es director de proyectos de investigación financiados por CONICET, ANPCyT y UNLP y ha participado en más de 34 proyectos desde 1980; cuenta con diversos libros publicados de diferentes temáticas como autor y co-autor, y con más de 170 publicaciones científicas de carácter internacional, regional y nacional. Ha asistido como expositor a múltiples congresos desde 1984. 